\title{
Uso da simulação como método no processo ensino-aprendizagem em saúde da criança: Revisão integrativa
}

\author{
Use of simulation as a method in the teaching-learning process in children's health: Integrative \\ review
}

Uso de la simulación como método en el proceso de enseñanza-aprendizaje en salud infantil: Revisión integradora

Viviane Correa Emiliano

ORCID: https://orcid.org/0000-0001-6675-6650 Instituto Federal de Educação, Ciência e Tecnologia do Paraná, Brasil E-mail: viviemiliano1@gmail.com

Nathália Borges da Costa Irineu ORCID: https://orcid.org/0000-0002-6299-6355 Instituto Federal de Educação, Ciência e Tecnologia do Paraná, Brasil E-mail: nathborges1703@gmail

Simone Roecker

ORCID: https://orcid.org/0000-0001-9812-720X Instituto Federal de Educação, Ciência e Tecnologia do Paraná, Brasil E-mail: simone.roecker@ifpr.edu.br

Adriana Martins Gallo

ORCID: https://orcid.org/0000-0003-0977-024X Instituto Federal de Educação, Ciência e Tecnologia do Paraná, Brasil E-mail: adriana.gallo@ifpr.edu.br

Adriana Valongo Zani

ORCID: https://orcid.org/0000-0002-6656-8155 Universidade Estadual de Londrina, Brasil E-mail: adrianazanienf@gmail.com

Juliane Pagliari Araujo

ORCID: https://orcid.org/0000-0001-7821-6731 Universidade Estadual de Londrina, Brasil E-mail: juliane.pagliari@ifpr.edu.br

\begin{abstract}
Resumo
Objetivo: identificar, na literatura científica, a utilização da simulação como método de ensino-aprendizagem, pela enfermagem, na área de saúde da criança. Metodologia: revisão integrativa, realizada nas bases de dados PUBMED e Biblioteca Virtual em Saúde, no período de 2010 a 2020, por meio de combinações entre os termos controlados e não controlados "simulation", "simulation training”, “children”, "nursing”, "nursing education”, com os operadores booleanos "and" e "or". Resultados: foram incluídos 13 estudos com simulação de alta e baixa fidelidade no cuidado à criança. Os estudos analisados apontam a eficácia do uso de simulação na assistência à criança, como metodologia ativa no processo ensino-aprendizagem e aquisição de habilidades, favorecendo o aprendizado, tanto dos estudantes como dos profissionais de saúde. Conclusão: o uso da simulação para o ensino da enfermagem, em específico, na área de saúde da criança, contribui de maneira eficaz para a qualificação profissional, nível de conhecimento, promoção do pensamento crítico, autoconfiança e comunicação interpessoal.
\end{abstract}

Palavras-chave: Simulação; Enfermagem; Aprendizagem interativa; Ensino de enfermagem; Saúde da criança.

\begin{abstract}
Objective: to identify, in the scientific literature, the use of simulation as a teaching-learning method by nursing in the area of child health. Methodology: integrative review, carried out in the PUBMED and Virtual Health Library databases, from 2010 to 2020, through combinations between the controlled and uncontrolled terms "simulation", "simulation training", "children", "nursing", "nursing education", with the Boolean operators "and" and "or". Results: 13 studies with simulation of high and low fidelity in child care were included. The analyzed studies point to the effectiveness of the use of simulation in child care, as an active methodology in the teaching-learning and skills acquisition process, favoring the learning of both students and health professionals. Conclusion: the use of simulation for teaching nursing, specifically, in the area of child health, effectively contributes to professional qualification, level of knowledge, promotion of critical thinking, self-confidence and interpersonal communication.
\end{abstract}


Keywords: Simulation; Nursing; Interactive learning; Nursing education; Child health.

\section{Resumen}

Objetivo: identificar, en la literatura científica, el uso de la simulación como método de enseñanza-aprendizaje por parte de la enfermería en el área de salud infantil. Metodología: revisión integradora, realizada en las bases de datos de PUBMED y Biblioteca Virtual en Salud, de 2010 a 2020, mediante combinaciones entre los términos controlados y no controlados "simulación", "entrenamiento en simulación", "niños", "enfermería", "educación en enfermería", con los operadores booleanos "and" y "or". Resultados: se incluyeron 13 estudios con simulación de alta y baja fidelidad en el cuidado infantil. Los estudios analizados apuntan a la efectividad del uso de la simulación en el cuidado infantil, como metodología activa en el proceso de enseñanza-aprendizaje y adquisición de habilidades, favoreciendo el aprendizaje tanto de los estudiantes como de los profesionales de la salud. Conclusión: el uso de la simulación para la enseñanza de enfermería, específicamente, en el área de la salud infantil, contribuye efectivamente a la calificación profesional, nivel de conocimientos, promoción del pensamiento crítico, autoconfianza y comunicación interpersonal.

Palabras clave: Simulación; Enfermería; Aprendizaje interactivo; Educación en enfermería; Salud de los niños.

\section{Introdução}

A formação em enfermagem é desafiadora para a transformação das práticas de saúde (Frota et al., 2020), e é essencial que seja de qualidade e que ofereça bagagem técnica-científica (Marcomini et al., 2017), para que o profissional tenha uma visão global interconectada com os avanços tecnológicos e culturais, garantindo, às populações, melhores indicadores de saúde e satisfação (Frota et al., 2020).

Neste cenário, a simulação realística vem ganhando espaço como um método de ensino-aprendizagem efetivo e inovador (Rodriguez et al., 2017), que amplia as relações entre a teoria e a prática do corpo discente em ambiente seguro, constrói situações semelhantes à realidade, oferece vivência do cotidiano profissional, treino de habilidades, comunicação, raciocínio clínico, análise e tomada de decisão, ensina o trabalho em equipe e melhora a autoconfiança, contribuindo para a formação profissional (Silva et al., 2020; Ferreira et al., 2018).

Os estudantes de enfermagem podem ser beneficiados pela estratégia de ensino que combina a teoria com a prática clínica, com foco na aprendizagem interativa, por meio da simulação para solucionar situações-problema, estimulando o pensamento crítico e reflexivo (Nascimento \& Magro, 2018; Kaneko \& Lopes, 2019; Silva et al., 2020).

Assim, a simulação realística é uma ferramenta que pode ser utilizada em todas as áreas de saúde e, ainda, ser aplicada em laboratório ou em ambiente real de trabalho, ou seja, "in-situ" (Alkhulaif et al., 2016), facilitando a interação entre a equipe multiprofissional assistencial e identificando as dificuldades e as possibilidades de melhoria tanto da infraestrutura, quanto da gestão do serviço (Saclabrini, Fonseca \& Brandão, 2017). O treinamento estruturado baseado em simulação e a avaliação de habilidades no trabalho são eficazes para melhorar a competência do cuidado (Hassanein et al., 2021).

Portanto, na enfermagem, a estratégia de ensino-aprendizagem com o uso de simulação está se tornando cada vez mais notória. Destaca-se a prática do cuidado de enfermagem em saúde da criança, que traz a simulação como uma forma de contribuição para mudanças do padrão de ensino tradicional, enriquecendo o conhecimento, aumentando a confiança dos estudantes e, consequentemente, aprimorando a qualidade do cuidado prestado (Fernandes et al., 2016).

Neste contexto, o objetivo do presente trabalho foi identificar, na literatura científica, a utilização da simulação como método de ensino-aprendizagem, pela enfermagem, na área de saúde da criança.

\section{Metodologia}

Trata-se de estudo do tipo revisão integrativa (Mendes, Silveira, Galvão, 2019; Souza et al, 2017) que se desenvolveu em seis etapas (Mendes, Silveira \& Galvão, 2019; Whittemore \& Knafl, 2005). Inicialmente, identificou-se o tema e formulouse a pergunta de pesquisa de acordo com a estratégia População Interesse Contexto (PICo) (Santos, Pimenta \& Nobre, 2014; Lockwood et al., 2020), considerando a seguinte estrutura: P- enfermagem e crianças, I- uso da simulação, Co- processo de 
ensino-aprendizagem. Dessa forma, elaborou-se a seguinte questão: Como a literatura tem evidenciado o uso da simulação pela enfermagem, no processo ensino-aprendizagem, na área da saúde da criança?

$\mathrm{Na}$ segunda etapa, estabeleceram-se os critérios para inclusão e exclusão dos estudos. Os critérios para inclusão foram artigos científicos, provenientes de estudos experimentais, descritivos ou analíticos, quantitativos e qualitativos, disponíveis na íntegra, que tratassem a temática e publicados nos últimos 10 anos (janeiro de 2010 a maio de 2020), visando obter os estudos mais recentes e relevantes, tendo também como foco o início do uso da simulação na área da enfermagem. Artigos que não fossem originais de pesquisa, além dos artigos duplicados e, outros gêneros textuais, foram considerados critério de exclusão.

Foram consultados os Descritores em Ciências da Saúde (DeCS) e definidos, para o presente estudo, os seguintes descritores: simulação, simulação realística, criança, ensino de enfermagem e enfermagem, e MeSH (Medical Subject Headings) "simulation", "simulation training”, “children", “nursing”, “nursing education". O cruzamento se deu pelos operadores booleano "and" e "or". Para conferir uma maior fidelidade ao estudo, as buscas e a seleção das produções científicas foram realizadas, de forma independente, por três revisores, e ocorreu no mês de junho de 2020. Foram utilizados artigos disponibilizados em modelo de publicação eletrônica na base de dados National Library of Medicine (PUBMED) e na Biblioteca Virtual em Saúde (BVS), a qual incluiu as bases Literatura Latino Americana e do Caribe em Ciências da Saúde (LILACS) e Medical Literature Analysis and Retrieval System OnLine (MEDLINE). A busca na PUBMED foi realizada por meio do portal de periódicos da Coordenação de Aperfeiçoamento de Pessoal de Nível Superior (CAPES), em área com Internet Protocol (IP) reconhecida no Instituto Federal do Paraná.

Identificaram-se 58 estudos, os quais passaram pela análise de critérios de inclusão previamente estabelecidos. A terceira etapa consistiu na leitura dos artigos selecionados, para identificar se respondiam à questão de pesquisa. Após essa leitura, os estudos foram selecionados para a presente revisão integrativa e fizeram parte da quarta etapa, que consistiu no preenchimento e avaliação do instrumento com as informações das publicações selecionadas. Para a coleta de dados, elaborouse instrumento que foi submetido à avaliação de três juízes, de forma independente e com experiência no tema investigado, e mais um instrumento contendo: autores, título, ano de publicação, periódico, local do estudo, objetivo, nível de evidência e desfecho.

A quinta etapa consistiu na discussão e interpretação dos resultados, momento em que foi realizada a comparação e a fundamentação dos principais achados com o conhecimento teórico, além da avaliação quanto a sua aplicabilidade. Seguida da sexta etapa, com a apresentação das evidências encontradas.

Para avaliação da qualidade metodológica, foi empregada a classificação de evidências, sendo: nível I - revisões sistemáticas com randomização; nível II - estudo clínico com randomização; nível III - estudo clínico sem randomização; nível IV - coorte e caso controle; nível V - revisão sistemática de estudos qualitativos; nível VI - estudos descritivos ou qualitativos; e nível VII - opinião de especialistas, descrição de casos (Melnyk \& Fineout-Overholt, 2019).

\section{Resultados}

Foram encontrados 58 artigos, dos quais, 34 oriundos da BVS e 24 da PUBMED. Após leitura do título e resumo, foram excluídos 39, por não atenderem aos critérios de inclusão na pesquisa e, após a leitura na íntegra, outros 06 também foram excluídos. A seleção foi finalizada com 13 artigos (Figura 1). 
Figura 1 - Fluxograma da seleção dos estudos para a revisão integrativa.

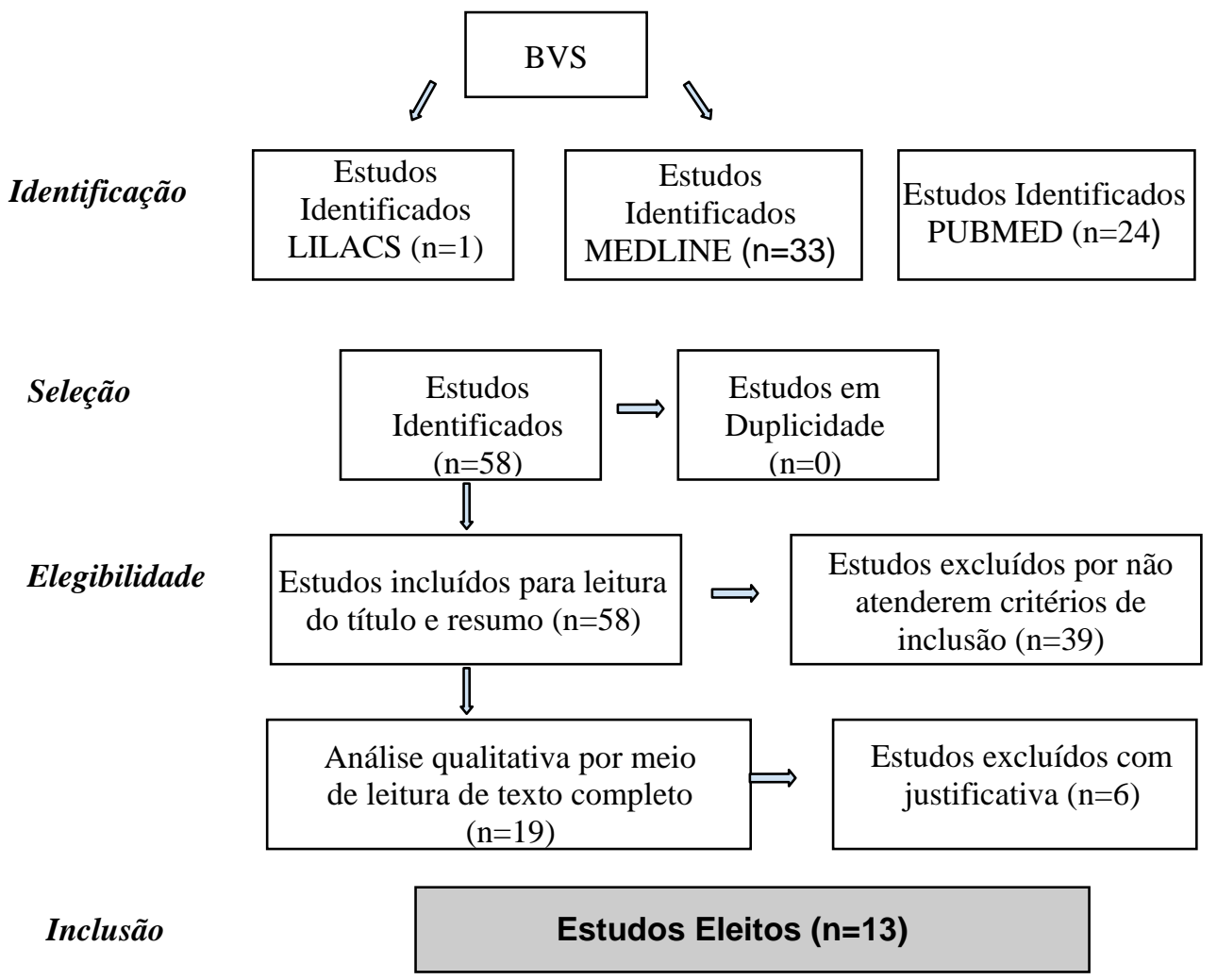

Fonte: Autores.

Foram selecionados 13 artigos que tiveram seus estudos desenvolvidos nos Estados Unidos da América (EUA) (n= 09), na Noruega ( $\mathrm{n}=01)$, no Canadá ( $\mathrm{n}=01)$, Austrália $(\mathrm{n}=01)$ e no Reino Unido $(\mathrm{n}=01)$, todos disponíveis em língua inglesa. No que se refere à abordagem dos estudos, 12 estudos eram quantitativos e um, qualitativo. Em análise ao nível de evidência dos artigos, observou-se que três estudos possuíam nível II, seis possuíam nível III, três estudos possuíam nível IV e um estudo era nível de evidência VI.

Dez estudos integraram, especificamente, a participação de enfermeiros e/ou acadêmicos em enfermagem, e três estudos de simulação de ensino, no ambiente profissional, também tiveram a participação de outros profissionais de saúde. Com relação ao cenário da simulação, seis estudos foram realizados no ambiente acadêmico e sete estudos no ambiente profissional.

As temáticas da saúde da criança abordadas foram: parada cardiorrespiratória $(n=4)$ e; vacinação, pós-operatório cardíaco, quimioterapia, obesidade, cuidados com cateter venoso central, doença cardíaca congênita, prática clínica de enfermagem, assistência na terminalidade de vida e relacionamento interpessoal $(n=1)$. A síntese dos estudos identificados, na presente revisão, foi apresentada no Quadro 1. 
Quadro 1 - Caracterização dos estudos das bases de dados consultadas, segundo autores, título, ano de publicação, país, periódico, objetivo, nível de evidência e desfecho. Brasil, 2020.

\begin{tabular}{|c|c|c|c|c|}
\hline Autor, título, ano & $\begin{array}{l}\text { País do } \\
\text { estudo; } \\
\text { periódico }\end{array}$ & Objetivo do estudo & $\begin{array}{c}\text { Nível } \\
\text { evidência }\end{array}$ & Desfecho \\
\hline $\begin{array}{l}\text { E1. Cole MA, et al. Simulação } \\
\text { pediátrica de fim de vida: } \\
\text { preparando o futuro enfermeiro } \\
\text { para cuidar das necessidades da } \\
\text { criança e da família (2018). }\end{array}$ & $\begin{array}{l}\text { EUA; } \\
\text { Journal of } \\
\text { Pediatric } \\
\text { Nursing }\end{array}$ & $\begin{array}{l}\text { Preparar o estudante para } \\
\text { responder às necessidades } \\
\text { complexas e sensíveis da } \\
\text { criança e sua família durante o } \\
\text { fim da vida. }\end{array}$ & III & $\begin{array}{l}\text { Aumentou a conscientização e a } \\
\text { sensibilidade quanto à } \\
\text { necessidade de comunicação com } \\
\text { a população infantil em estado } \\
\text { terminal. }\end{array}$ \\
\hline $\begin{array}{l}\text { E2. Akselbo I, et al. Simulação } \\
\text { como método de aprendizagem na } \\
\text { formação de enfermeiros em } \\
\text { saúde pública (2018). }\end{array}$ & $\begin{array}{l}\text { Noruega; } \\
\text { Public Health } \\
\text { Nursing }\end{array}$ & $\begin{array}{l}\text { Verificar a simulação como um } \\
\text { método de aprendizado à luz } \\
\text { das teorias da aprendizagem e } \\
\text { descrever as reflexões e } \\
\text { experiências do estudante em } \\
\text { simulação de anafilaxia durante } \\
\text { a vacinação. }\end{array}$ & III & $\begin{array}{l}\text { Os estudantes de enfermagem } \\
\text { experimentaram a simulação } \\
\text { como um método educacional } \\
\text { realista e eficaz. }\end{array}$ \\
\hline $\begin{array}{l}\text { E3. Brown KM, et al. Campo de } \\
\text { simulação multi-institucional para } \\
\text { profissionais de enfermagem de } \\
\text { cuidados cardíacos intensivos em } \\
\text { pediatria (2018). }\end{array}$ & $\begin{array}{l}\text { EUA; } \\
\text { Pediatric } \\
\text { Critical Care } \\
\text { Medicine }\end{array}$ & $\begin{array}{l}\text { Avaliar a capacidade dos } \\
\text { enfermeiros pediátricos em } \\
\text { identificar e tratar uma } \\
\text { diminuição do débito cardíaco } \\
\text { em crianças no pós-operatório } \\
\text { com doença cardíaca. }\end{array}$ & III & $\begin{array}{l}\text { Melhorou o conhecimento e os } \\
\text { profissionais relataram satisfação } \\
\text { e confiança na experiência de } \\
\text { simulação. }\end{array}$ \\
\hline $\begin{array}{l}\text { E4. Gilfoyle E, et al. Desempenho } \\
\text { clínico e trabalho em equipe das } \\
\text { equipes pediátricas de } \\
\text { ressuscitação interprofissional } \\
\text { aprimorados com uma intervenção } \\
\text { educacional baseada em } \\
\text { simulação (2017). }\end{array}$ & $\begin{array}{l}\text { Canadá; } \\
\text { Online } \\
\text { Clinical } \\
\text { Investigation }\end{array}$ & $\begin{array}{l}\text { Medir o efeito de um } \\
\text { treinamento para membros da } \\
\text { equipe de ressuscitação } \\
\text { interprofissional pediátrica. }\end{array}$ & III & $\begin{array}{l}\text { Apresentou uma correlação } \\
\text { positiva entre desempenho clínico } \\
\text { e trabalho em equipe. Sugere que } \\
\text { o trabalho em equipe é eficaz e } \\
\text { melhora o desempenho clínico da } \\
\text { ressuscitação pediátrica. }\end{array}$ \\
\hline $\begin{array}{l}\text { E5. Sawyer T, et al. Treinamento } \\
\text { de ventilação com pressão positiva } \\
\text { durante a ventilação com bolsa- } \\
\text { máscara neonatal: um estudo } \\
\text { piloto baseado em simulação } \\
\text { (2019). }\end{array}$ & $\begin{array}{l}\text { EUA; } \\
\text { Journal of } \\
\text { Neonatal- } \\
\text { Perinatal } \\
\text { Medicine }\end{array}$ & $\begin{array}{l}\text { Avaliar os benefícios do } \\
\text { treinamento com ventilação de } \\
\text { pressão positiva (VPP) durante } \\
\text { a ventilação com bolsa-máscara } \\
\text { neonatal. }\end{array}$ & II & $\begin{array}{l}\text { O treinamento melhorou } \\
\text { desempenho da } \\
\text { simulação. }\end{array}$ \\
\hline $\begin{array}{l}\text { E6. Linnard-Palmer L. O uso de } \\
\text { simulação para princípios de } \\
\text { segurança de enfermagem } \\
\text { oncológica pediátrica: garantindo } \\
\text { prática competente através do uso } \\
\text { de um roteiro mnemônico de } \\
\text { quimioterapia e aprendizagem } \\
\text { baseada em casos (2012). }\end{array}$ & $\begin{array}{l}\text { EUA; } \\
\text { SPN News }\end{array}$ & $\begin{array}{l}\text { Fornecer um seminário } \\
\text { educacional com cenário de } \\
\text { simulação de fidelidade para } \\
\text { aprender, praticar e aplicar os } \\
\text { componentes essenciais de } \\
\text { segurança do tratamento } \\
\text { durante os cuidados de uma } \\
\text { criança com câncer. }\end{array}$ & IV & $\begin{array}{l}\text { Apontou ganho de confiança, } \\
\text { satisfação e aquisição de } \\
\text { habilidades. A simulação foi } \\
\text { percebida como ótimo método de } \\
\text { aprendizado, quanto à segurança } \\
\text { na administração de } \\
\text { medicamentos quimioterápicos. }\end{array}$ \\
\hline $\begin{array}{l}\text { E7. Kolko RP, et al. Treinamento } \\
\text { para rastrear e iniciar o tratamento } \\
\text { da obesidade pediátrica com base } \\
\text { em evidências em ambientes de } \\
\text { prática de rotina: um ensaio piloto } \\
\text { randomizado (2017). }\end{array}$ & $\begin{array}{l}\text { EUA; } \\
\text { Journal of } \\
\text { Pediatric } \\
\text { Health Care }\end{array}$ & $\begin{array}{l}\text { Avaliar duas modalidades de } \\
\text { treinamento, online e ao vivo, } \\
\text { entre estudantes de } \\
\text { enfermagem, baseadas em } \\
\text { evidências em um serviço de } \\
\text { obesidade pediátrica. }\end{array}$ & II & $\begin{array}{l}\text { Esses treinamentos, em larga } \\
\text { escala, tanto no meio acadêmico, } \\
\text { quanto no ambiente profissional, } \\
\text { podem otimizar os resultados e } \\
\text { promover melhor prestação de } \\
\text { cuidados. }\end{array}$ \\
\hline $\begin{array}{l}\text { E8. Hebbar KB, et al. } \\
\text { Treinamentos baseados em } \\
\text { simulação para manutenção de } \\
\text { cateter venoso central em unidade } \\
\text { de terapia intensiva pediátrica } \\
(2014) \text {. }\end{array}$ & $\begin{array}{l}\text { EUA; } \\
\text { Intensive and } \\
\text { Critical Care } \\
\text { Nursing }\end{array}$ & $\begin{array}{l}\text { Melhorar a manutenção, } \\
\text { agrupar taxas de conformidade } \\
\text { e competência de enfermagem } \\
\text { em torno dos cuidados com } \\
\text { cateter venoso central em uma } \\
\text { Unidade de Terapia Intensiva } \\
\text { (UTI) pediátrica. }\end{array}$ & II & $\begin{array}{l}\text { A simulação demonstrou maior } \\
\text { efeito se comparado com a prática } \\
\text { de treinamento padrão com } \\
\text { evidência de melhoria na retenção } \\
\text { de conhecimento de habilidades. }\end{array}$ \\
\hline
\end{tabular}




\begin{tabular}{|c|c|c|c|c|}
\hline $\begin{array}{l}\text { E9. Searl KR, et al. Pessoas } \\
\text { pequenas, grandes lições: Uma } \\
\text { estratégia inovadora para } \\
\text { desenvolver o relacionamento } \\
\text { interpessoal e habilidades em } \\
\text { estudantes de graduação em } \\
\text { enfermagem (2014). }\end{array}$ & $\begin{array}{l}\text { Austrália; } \\
\text { Nurse } \\
\text { Education } \\
\text { Today }\end{array}$ & $\begin{array}{l}\text { Buscar informações de } \\
\text { estudantes de Enfermagem, por } \\
\text { meio do uso de fantoches sob a } \\
\text { luz da teoria interpessoal, sobre } \\
\text { a aceitação, capacidade e } \\
\text { impacto da simulação. }\end{array}$ & VI & $\begin{array}{l}\text { Aprofundou percepções sobre o } \\
\text { processo educativo e os impactos } \\
\text { de aprendizagem, o qual sugerem } \\
\text { que a aprendizagem baseada em } \\
\text { fantoches é um meio para unir } \\
\text { teoria e prática. }\end{array}$ \\
\hline $\begin{array}{l}\text { E10. Campbell JM. Educação e } \\
\text { simulação de treinamento de } \\
\text { enfermeiros de unidade de terapia } \\
\text { intensiva pediátrica r para } \\
\text { atendimento de pacientes } \\
\text { submetidos a cirurgia cardíaca } \\
\text { aberta (2015). }\end{array}$ & $\begin{array}{l}\text { EUA; } \\
\text { Critical Care } \\
\text { Nurse }\end{array}$ & $\begin{array}{l}\text { Desenvolver e atualizar o } \\
\text { conjunto de habilidades e } \\
\text { conhecimentos necessários para } \\
\text { cuidar de bebês e crianças com } \\
\text { doença cardíaca congênita. }\end{array}$ & IV & $\begin{array}{l}\text { As experiências foram positivas. } \\
\text { O conjunto de habilidades } \\
\text { necessário para reagir em } \\
\text { condições críticas foram } \\
\text { desenvolvidas por meio de } \\
\text { experiências praticadas. }\end{array}$ \\
\hline $\begin{array}{l}\text { E11. Davies J, et al. Uma } \\
\text { avaliação de um cenário simulado } \\
\text { complexo com alunos do último } \\
\text { ano de graduação em enfermagem } \\
\text { infantil (2012). }\end{array}$ & $\begin{array}{l}\text { Reino Unido; } \\
\text { Collegian }\end{array}$ & $\begin{array}{lcr}\begin{array}{l}\text { Avaliar a } \\
\text { simulação }\end{array} \text { experiência } & \text { dánica } \\
\text { Enfermagem } & \text { infantil } \\
\text { método de aprendizagem. } & \end{array}$ & IV & $\begin{array}{l}\text { O uso de simulação pode fornecer } \\
\text { aos estudantes uma experiência } \\
\text { segura para prática profissional. }\end{array}$ \\
\hline $\begin{array}{l}\text { E12. Kane J, et al. Eficácia de um } \\
\text { programa educacional baseado em } \\
\text { simulação em uma unidade de } \\
\text { terapia intensiva cardíaca } \\
\text { pediátrica (2011). }\end{array}$ & $\begin{array}{l}\text { EUA; } \\
\text { Journal of } \\
\text { Pediatric } \\
\text { Nursing }\end{array}$ & $\begin{array}{l}\text { Desenvolver e implementar um } \\
\text { novo método de treinamento } \\
\text { para preparar o enfermeiro } \\
\text { pediátrico da UTI para realizar } \\
\text { ressuscitação eficaz em bebês e } \\
\text { crianças com doença cardíaca } \\
\text { congênita. }\end{array}$ & III & $\begin{array}{l}\text { É um método eficaz de educar a } \\
\text { equipe de enfermagem com } \\
\text { conhecimentos e habilidades } \\
\text { necessários para a reanimação. O } \\
\text { feedback foi positivo, e o } \\
\text { desempenho das reanimações } \\
\text { reais melhorou. }\end{array}$ \\
\hline $\begin{array}{l}\text { E13. Van Schaik SM, et al. } \\
\text { Treinamento da equipe } \\
\text { interprofissional em ressuscitação } \\
\text { pediátrica: um programa de } \\
\text { simulação in situ de baixo custo } \\
\text { que aumenta a autoeficácia entre } \\
\text { os participantes (2011). }\end{array}$ & $\begin{array}{l}\text { EUA; } \\
\text { Clinical } \\
\text { Pediatrics }\end{array}$ & $\begin{array}{llr}\text { Realizar uma análise } & \text { do } \\
\text { impacto do programa } & \text { de } \\
\text { treinamento, baseado } & \text { em } \\
\text { simulação in situ } & \text { de } \\
\text { emergências pediátricas } & \mathrm{e} \\
\text { ressuscitação. } & & \end{array}$ & III & $\begin{array}{l}\text { Ilustrou como um processo } \\
\text { colaborativo entre diferentes } \\
\text { profissionais pode levar a um } \\
\text { programa sustentável que engloba } \\
\text { a segurança do paciente. }\end{array}$ \\
\hline
\end{tabular}

Fonte: Autores.

\section{Discussão}

Os estudos analisados destacam a eficácia do uso de simulação na assistência à criança, como metodologia ativa no processo ensino-aprendizagem e aquisição de habilidades, favorecendo o aprendizado, tanto dos estudantes, como dos profissionais de saúde.

A simulação é um importante método, utilizado tanto como processo de aprendizagem, quanto de avaliação (Costa et al., 2020). Com suas características metodológicas, as práticas simuladas na enfermagem contribuem efetivamente para a formação e promovem a autoconfiança, autonomia e satisfação (Costa et al., 2020, Baptista et al., 2014, Brown et al., 2018, Linnard-Palmer, 2012). Ao participar ativamente das atividades de simulação, o estudante passa a ampliar seu senso crítico e aperfeiçoar seu modo de analisar as diferentes situações impostas (Costa et al., 2017). O uso da simulação no cuidado infantil aumentou a sensibilidade quanto à necessidade de comunicação com essa população (Cole \& Foito, 2018), assim como a empatia é fundamental para ganhar a credibilidade de uma criança e ajudá-la a sentir-se confortável e segura em um ambiente de saúde (Searl et al., 2014).

Dentre as simulações, qualifica-se a alta, média e baixa fidelidade, sendo que cada modalidade tem seu grau de aproximação com a realidade. A escolha do tipo de simulação deve estar vinculada com o objetivo e complexidade do cuidado dispensado à criança. Os simuladores de alta fidelidade apresentam um alto grau de aproximação com a realidade, a exemplo do paciente computadorizado, interativo, com respiração e respostas fisiopatológicas próximas às reais, diferente dos 
simuladores de média fidelidade que apresentam sons pulmonares e cardíacos e, ainda, de baixa fidelidade com manequins estáticos, sem interação (Bortolato-Major et al., 2018, Lacerda et al., 2020).

Dentre os artigos eleitos, 12 trabalharam com simuladores de alta fidelidade, sendo destacada a oportunidade de discutir quaisquer preocupações ou medos, autoconsciência, bem como, desenvolver habilidades técnicas no cuidado à criança (Kolko et al., 2017, Hebbar et al., 2015, Campbell, 2015). Apenas um estudo eleito utilizou simulador de baixa fidelidade, destacando que o treinamento de simulação foi útil e impactou positivamente seus conhecimentos, conforto e habilidades no manejo ao grupo infantil (Searl et al., 2014). Outro estudo realizado com simulação de baixa fidelidade, relatando a experiência de discentes, destacou a importância da simulação no ensino da enfermagem (Molonha et al., 2020). Nesse sentido, tem-se que a simulação, independentemente do nível de fidelidade (alta, média ou baixa) tem reflexo positivo como estratégia no processo de ensino-aprendizagem na área da saúde da criança.

Destaca-se que o docente tem autonomia para orientar e organizar o modo de ensinar determinadas práticas clínicas no cuidado à criança. O uso misto de métodos também mostrou-se eficaz (Aldridge, 2019). No entanto, ainda são necessários mais estudos que abordem a temática da docência em ensino de simulação realística, para auxiliar na formação dos futuros profissionais docentes de enfermagem, com base na mais alta tecnologia e inovação, a fim de garantir qualidade no processo ensino-aprendizagem e, ao mesmo tempo, segurança da criança, da família e excelência profissional (Carneiro et al., 2019).

A importância do trabalho em equipe multiprofissional tem sido cada vez mais reconhecida, porém, o treinamento uniprofissional com atividades direcionadas a estudantes da mesma profissão ainda acontecem em grande escala (Khan, Shahnaz \& Gomathi, 2016). Assim, faz-se necessária a integração de professores das diferentes áreas em ambiente simulado, pois essa integração pode potencializar a compreensão dos estudantes e profissionais de saúde acerca da atenção integral à criança e sua família (Fonseca et al., 2020). Nesse contexto, várias estratégias de ensino têm sido propostas para esta finalidade, como atividades de promoção da saúde, treinamentos multiprofissionais em enfermarias, estudos de caso e simulações com cenários interprofissionais (Khan, Shahnaz \& Gomathi, 2016). Profissionais de saúde que participaram de simulações multiprofissionais valorizaram a natureza interprofissional do treinamento (Kane, Pye \& Jones, 2011, Van Schaik et al., 2011). No entanto, a simulação em ambiente profissional ainda demanda de alguns desafios como: definição de tipo de treinamento (alta ou baixa fidelidade), tempo e motivação para a equipe participar, além do horário de trabalho, custo das sessões de simulação e com o educador (Sawyer et al., 2019).

Em um olhar aprofundado na literatura científica nacional e internacional, é possível observar que diversos estudos apresentam evidências de que a simulação, no processo de formação de profissionais em pediatria e neonatologia, consequentemente envolvidos com a saúde da criança, contribui para o desenvolvimento de habilidades clínicas e de procedimentos (Bowling, 2015, Stephenson, Salih \& Cullen, 2015, Valizadeh et al., 2013, Linnard-Palmer, 2012, Gilfoyle et al., 2017, Kolko et al., 2017), melhora o nível de conhecimento (Barracho, Chaves \& Lucas, 2020, Brown et al., 2018, Mesquita, Santana \& Magro, 2019) e a satisfação (Brown et al., 2018, Mesquita, Santana \& Magro, 2019), promove o pensamento crítico (Valizadeh et al., 2013; Brown et al., 2018, Gilfoyle et al., 2017) e ainda, favorece a autoconfiança e comunicação interpessoal (Shin, Park \& Kim, 2015, Brown et al., 2018, Linnard-Palmer, 2012, Akselbo, et al., 2018, Gilfoyle et al., 2017; Bellaguarda et al., 2020).

A prática simulada no âmbito das ferramentas digitais para a formação em saúde apresenta vantagens, entre elas está a garantia da segurança do paciente (Fonseca et al., 2016; Molonha et al., 2020, Davies et al., 2015), pois o estudante não terá sua primeira experiência prática diretamente com um paciente, e a ética na assistência, uma vez que os estudantes estarão mais bem preparados para as situações reais, depois da simulação prévia. Considerando que nem todas as situações de intervenção em saúde podem ser realizadas pelos estudantes, como situações de urgência, reforçam-se os benefícios de aprendizagem presentes, ao viabilizar ao estudante a experiência simulada (Fonseca et al., 2016; Martins et al., 2012). No entanto, destaca-se 
que o contato direto com o paciente é de extrema importância para a formação profissional.

$\mathrm{Na}$ área da educação, no ano de 2020, o mundo precisou de adaptações, devido à pandemia de COVID-19, causada pelo novo Coronavírus. Nesse contexto e, especificamente sobre o ensino de enfermagem, existem muitas restrições com relação à exposição de estudantes durante as atividades ligadas ao atendimento de pacientes. Um estudo realizado na Coreia do Sul apontou que estratégias educacionais eficazes são necessárias para aumentar a competência dos estudantes na prática clínica, utilizando a simulação e a prática baseada em evidências, avaliando a aprendizagem por meio do uso operacional de simulações e aumentando o desempenho de habilidades básicas, além de ter demonstrado a satisfação dos estudantes (Son, 2020).

A utilização de métodos mútuos de ensino deve ser levada em consideração, vislumbrando-se que a simulação motiva os estudantes a despertarem e modificarem alguns vícios de comportamento, que poderiam prejudicar o estado de saúde do paciente, no entanto, não devem substituir a prática profissional que deve ser conciliada (Beserra et al., 2020).

Tendo em vista que, na presente revisão de literatura, nenhum dos estudos que compuseram a amostra possui nível I e apenas três estudos possuem nível II de evidência, percebe-se a necessidade de pesquisas que produzam resultados com altos níveis de evidência científica, a fim de subsidiar o ensino, por meio da simulação realística no contexto infantil. Torna-se imperativo fomentar a discussão sobre o uso da simulação no ensino da saúde da criança, bem como instigar a construção do conhecimento a partir novos estudos que tragam evidências científicas e diferentes experiências.

\section{Conclusão}

O uso da simulação para o ensino-aprendizagem na enfermagem, em específico, na área de saúde da criança, contribui para a qualificação profissional, com destaque para as habilidades clínicas e em procedimentos.

Os resultados do presente estudo evidenciam uma melhora do nível de conhecimento para a promoção do pensamento crítico, bem como favorecem a autoconfiança e comunicação interpessoal. A eficácia da prática da simulação, pautada em evidências, enriquece o ensino da enfermagem em saúde da criança.

\section{Referências}

Akselbo, I., Olufsen, V., Ingebrigtsen, O., \& Aune, I. (2019). Simulation as a learning method in public health nurse education. Public Health Nurs, 36(2):1-7. https://doi.org/10.1111/phn.12560

Aldridge, M. D. (2019). Teaching nursing students the care of a patient with end stage renal disease in a pediatric nursing theory course: a comparison of two instructional methods. Nephrol Nurs J. 46(6):605-610. https://pubmed.ncbi.nlm.nih.gov/31872991

Alkhulaif, A., Julie, I., Barton, J., Nagle, E., Yao, A., Clarke S, et al. (2016) In-situ simulation training: advantages, challenges and obstacles. Latin Am J Telehealth. 3(2):141-149. http://cetes.medicina.ufmg.br/revista/index.php/rlat/article/view/139/279

Baptista, R. C. N., Martins, J. C. A., Pereira, M. F. C. R. \& Mazzo, A. (2014) Students' satisfaction with simulated clinical experiences: validation of an assessment scale. Rev. Latino-Am Enfermagem. 22(5):709-715. http://dx.doi.org/10.1590/0104-1169.3295.2471

Baracho, V. S., Chaves, M. E. A, \& Lucas, T. C. (2020). Application of the educational method of realistic simulation in the treatment of pressure injuries. Rev Latino-Am Enfermagem. 28(e3357);1-8. https://doi.org/10.1590/1518-8345.3946.3357

Bellaguarda, M. L. R., Knihs, N. S., Canever, B. P., Tholl, A. D., Alvarez, A. G., \&Teixeira, G. C. (2020). Realistic simulation as a teaching tool in critical situation communication in palliative care. Esc Anna Nery. 24(3):1-8. http://dx.doi.org/10.1590/2177-9465-ean-2019-0271

Beserra, E. P., Camelo, L. B. M., Teles, L. M. R., Barbosa, J. E. C., Cavalcante, V. M. V., Gubert, F.A., et al. (2020) Realistic simulation in immunization: satisfaction, self-confidence and performance of nursing students. Rev RENE. [Internet]. 21(e44514):1-8. https://doi.org/10.15253/2175-6783.20202144514

Bortolato-Major, C., Arhur, J. P., Silva, Â. T. M., Mantovani, M. F., Felix, J. V. C., \& Boostel, R. (2018) Contributions of the simulation for undergraduate nursing students. Rev Enferm UFPE. 12(6):1751-1762. https://doi.org/10.5205/1981-8963-v12i6a230633p1751-1762-2018

Bowling, A. M. (2015) The effect of simulation on skill performance: a need for change in pediatric nursing education. J Pediatr Nurs. 30(3):439-446. https://doi.org/10.1016/j.pedn.2014.12.010

Brown, K. M., Mudd, S. S., Hunt, E. A., Perretta, J. S., Shilkofski, N. A., Diddlem, J. W., et al. (2018). A multi-institutional simulation boot camp for pediatric cardiac critical care nurse practitioners. Pediatr Crit Care Med. 19(6):564-571. https://doi.org/10.1097/PCC.0000000000001532 
Campbell, J. M. (2015). Education and simulation training of pediatric intensive care unit nurses to care for open heart surgery patients. Crit Care Nurse. 35(3):76-81. https://doi.org/10.4037/ccn2015312

Carneiro, K. K. C., Moraes-Filho, I. M., Santos, O. P., Arantes, A. A., Félis, K. C., Guilherme, I. S., et al. (2019) Simulação realística como instrumento no processo de ensino-aprendizagem de enfermagem. Revisa: Revista Bras Vigil Sanit. 8(3):273-284. https://doi.org/10.36239/revisa.v8.n3.p273a284

Cole, M. A. \& Foito, K. (2018). Pediatric end-of-life simulation: preparing the future nurse to care for theneeds of the child and family. J Pediatr Nurs. 44:e9e12. https://doi.org/10.1016/j.pedn.2018.09.005

Costa, R. R. O., Medeiros, S., Martins, J. C. \& Cossi, M. (2017). Percepção de estudantes da graduação em enfermagem sobre a simulação realística. Rev Cuid. 8(3):1799-1808. http://dx.doi.org/10.15649/cuidarte.v8i3.425

Costa, R. R. O., Medeiros, S. M. M., Martins, J. C. A. M., Coutinho, V. R. D., \& Araújo, M. S. A. (2020). Effectiveness of simulation in teaching immunization in nursing: a randomized clinical trial. Rev Latino-Am Enfermagem. 28e3305, 2020. https://doi.org/10.1590/1518-8345.3147.3305

Davies, J., Nathan, M., \& Clarke, D. (2012). An evaluation of a complex simulated scenario with final year undergraduate children's nursing students. Collegian. 19(3):131-138. https://doi.org/10.1016/j.colegn.2012.04.005

Fernandes, A. K. C., Ribeiro, L. M., Brasil, G. C., Magro, M. C. S., Hermann, P. R. S., Ponce de Leon, C. G. R. M., et al. (2016). Simulation as a strategy for learning in pediatrics. Rev Min Enferm. 20(976):1-7. http://www.dx.doi.org/10.5935/1415-2762.20160046

Ferreira, R. P., Guedes, H. M., Oliveira, D. W. D., \& Miranda, J. L. (2018). Realistic simulation as a method of teaching in the learning of the health field students. $R$ Enferm Cent O Min. 8. https://doi.org/10.19175/recom.v8i0.2508

Fonseca, L. M. M., Monteiro, J. C. S., Aredes, N. D. A., Bueno, J. V., Domingues, A. N., Coutinho, V. R. D., et al. (2020). Interdisciplinary simulation scenario in nursing education: Humanized childbirth and birth. Rev Latino-Am Enfermagem. 28:(e3286). https://doi.org/10.1590/1518-8345.3681.3286

Frota, M. A., Wermelinger, M. C. M. W., Vieira, L. J. E. S., Ximenes Neto, F. R. G., Queiroz, R. S. M., \& Amorim, R. F. (2020). Mapping nursing training in Brazil: challenges for actions in complex and globalized scenarios. Ciênc Saúde Colet. 25(1):25-35. https://doi.org/10.1590/1413-81232020251.27672019

Gilfoyle, E., Koot, D. A., Annear, J. C., Bhanji, F., Cheng, A., Duff, J. P., et al. (2017). Improved clinical performance and teamwork of pediatric interprofessional resuscitation teams with a simulation-based educational intervention. Pediatr Crit Care Med. 18(2):e62-e69. https://doi.org/10.1097/PCC.0000000000001025

Hassanein, S. M. A., Tantawi, H. R., Sadek, B. N., Hendy, A., \& Awad, H. A. (2021). Impact of structured simulation-based and on-job training program on nurses' competency in pediatric peripheral intravenous cannulation: children's hospital experience. Nurse Educ Today. 98(104776). https://doi.org/10.1016/j.nedt.2021.104776

Hebbar, K. B., Cunningham, C., McCracken, C., Kamat, P., \& Fortenberry, J. D. (2015). Simulation-based paediatric intensive care unit central venous line maintenance bundle training. Intensive Crit Care Nurs. 31(1):44-50. http://dx.doi.org/10.1016/j.iccn.2014.10.003

Kane, J., Pye, S., \& Jones, A. (2011). Effectiveness of a simulation-based educational program in a pediatric cardiac intensive care unit. J Pediatr Nurs. 26(4):287-294. https://doi.org/10.1016/j.pedn.2010.05.004

Kaneko, R. M. U., \& Lopes, M. H. B. M. (2019). Realistic health care simulation scenario: what is relevant for its design? Rev Esc Enferm USP [Internet]. 53(e03453):1-8. https://doi.org/10.1590/s1980-220x2018015703453

Khan, N. S., Shahnaz, S. I., \& Gomathi, K. G. (2016). Currently available tools and teaching strategies for the interprofessional education of students in health professions. Sultan Qaboos Univ Med J. 16(3):277-285. https://doi.org/10.18295/squmj.2016.16.03.003

Kolko, R. P., Kass, A. E., Hayes, J. F., Levine, M. D., Garbutt, J. M., Proctor, E. K., et al. (2017). Provider training to screen and initiate vidence-based pediatric obesity treatment in routine practice settings: a randomized pilot trial. J Pediatr Health Care. 31(1):16-28. https://doi.org/10.1016/j.pedhc.2016.01.001

Lacerda, C. S., Sá, S. P. C., Braga, A. L. S., Balbino, C. M., \& Silvino, Z. R. (2020). Simulation as an active methodology for the education of students in nursing: an integrative review. Online Braz J Nurs. 19(2):1-16. https://doi.org/10.17665/1676-4285.20206490

Linnard-Palmer, L. (2012). The use of simulation for pediatric oncology nursing safety principles: ensuring competent practice through the use of a mnemonic, chemotherapy road maps and case-based learning. J Pediatr Nurs. 27(3):283-286. https://doi.org/10.1016/j.pedn.2012.02.001

Lockwood, C., Porrit, K., Munn, Z., Rittenmeyer, L., Salmond, S., Bjerrum, M., et al. (2020). Systematic reviews of qualitative evidence. In: Aromataris E, Munn Z, editors. JBI manual for evidence synthesis. Adelaide: JBI. https://doi.org/10.46658/JBIMES-20-03

Marcomini, E. K., Martins, E. S. M., Lopes, N. V., Paula, N. V. K., \& Liberati, B. A. S. (2017). Influência da simulação realística no ensino e aprendizado da enfermagem. Varia Sci. 3(2):233-240. https://doi.org/10.48075/vscs.v3i2.17687

Martins, J. C. A., Mazzo, A., Baptista, R. C. N., Coutinho, V. R. D., Godoy, S., Mendes, I. A. C., et al. (2012). The simulated clinical experience in nursing education: a historical review. Acta Paul Enferm. 25(4):619-625. https://doi.org/10.1590/S0103-21002012000400022

Melnyk, B. M. \& Fineout-Overholt, E. (2019). Evidence-based practice in nursing and healthcare: a guide to best practice. Wolters Kluwer.

Mendes, K. D. S., Silveira, R. C. C. P., \& Galvão, C. M. (2019). Use of the bibliographic reference manager in the selection of primary studies in integrative reviews. Texto Contexto Enferm 28:1-13. https://dx.doi.org/10.1590/1980-265X-TCE-2017-0204

Mesquita, H. C. T., Santana, B. S., \& Magro, M. C. S. (2019). Effect of realistic simulation combined to theory on self-confidence and satisfaction of nursing professionals. Esc Anna Nery. 23(1):1-6. https://doi.org/10.1590/2177-9465-ean-2018-0270 
Research, Society and Development, v. 10, n. 9, e30810917999, 2021

(CC BY 4.0) | ISSN 2525-3409 | DOI: http://dx.doi.org/10.33448/rsd-v10i9.17999

Molonha, A, Fakhr, B. O., Custódio, F. A., Lopes, T. P., Roecker, S., \& Araujo, J. P. (2020). Simulation as a teaching-learning strategy in wound treatment: experience report. Rev Baiana Enferm. 34(e37039):1-6. http://dx.doi.org/10.18471/rbe.v34.37039

Nascimento, M. S., \& Magro, M. C. S. (2018). Realistic simulation: method of improving knowledge and self-confidence of nursing students in the administration of medication. Rev Min Enferm. 22(e-1094):1-5. http://www.dx.doi.org/10.5935/1415-2762.20180024

Rodriguez, K. G., Nelson, N., Gilmartin, M., Goldsant, L., \& Richardson, H. (2017). Simulation is more than working with a mannequin: student's perceptions of their learning experience in a clinical simulation environment. J Nurs Educ Pract. 7(7):30-36. https://doi.org/10.5430/jnep.v7n7p30

Saclabrini, A., Fonseca, A., \& Brandão, C. (2017). Simulação realística e habilidades na saúde. Atheneu.

Santos, C. M. C., Pimenta, C. A. M., \& Nobre, M. R. C. (2014). The PICO strategy for the research question construction and evidence search. Rev Lat Am Enfermagem. [Internet]. 15(3):508-11. https://doi.org/10.1590/S0104-11692007000300023

Sawyer, T., Motz, P., Schooley, N., \& Umoren, R. (2019). Positive pressure ventilation coaching during neonatal bag-mask ventilation: a simulation-based pilot study. J Neonatal Perinatal Med. 12(3):243-248. https://doi.org/10.3233/NPM-18119

Searl, K. R., McAllister. M., Dwyer, T., Krebs, K. L., Quinney, C. A. L., \& McLellan, S. (2014). Little people, big lessons: an innovative strategy to develop interpersonal skills in undergraduate nursing students. Nurse Educ Today 34(9):1201-1206. https://doi.org/10.1016/j.nedt.2014.04.004

Shin, S., Park, J-H.,\& Kim J-H. (2015). Effectiveness of patient simulation in nursing education: meta-analysis. Nurse Educ Today. 35(1):176-182. http://dx.doi.org/10.1016/j.nedt.2014.09.009

Silva, R. P., Santos, V. S., Moraes, J. S., Andrade, I. R. C., Abreu, R. N. D. C., \& Freitas, J. G. (2020). Applicability of realistic simulation in nursing graduation: experience in incidents involving multiple victims. Rev Baiana Enferm. 34(34648):1-11. http://dx.doi.org/10.18471/rbe.v34.34648

Son, H. K. (2020). Effects of S-PBL in maternity nursing clinical practicum on learning attitude, metacognition, and critical thinking in nursing students: a quasi-experimental design. Int J Environ Res Public Health. 17(21):1-12. https://doi.org/10.3390/ijerph17217866

Stephenson, E., Salih, Z., \& Cullen, D. L. (2015). Advanced practice nursing simulation for neonatal skill competency: a pilot study for successful continuing education. J Contin Educ Nurs. 46(7):322-325. http://dx.doi.org/10.3928/00220124-20150619-04

Valizadeh, L., Amini, A., Fathi-Azar, E., Ghiasvandian, S., \& Akbarzadeh, B. (2013). The effect of simulation teaching on baccalaureate nursing students selfconfidence related to peripheral venous catheterization in children: a randomized trial. J Caring Sci. 2(2):157-164. https://doi.org/10.5681/jcs.2013.019

Van Schaik, S. M., Plant, J., Diane, S., Tsang, L., \& O’Sullivan, P. (2011). Interprofessional team training in pediatric resuscitation: a low-cost, in situ simulation program that enhances self-efficacy among participants. Clin Pediatr. 50(9):807-815. https://doi.org/10.1177/0009922811405518

Whittemore, R., \& Knafl, K. (2005). The integrative review: updated methodology. J Adv Nurs. 52(5):546-553. 10.1111/j.1365-2648.2005.03621.x 